\title{
A Model of a Wireless Factory Work-Cell Using the Systems Modeling Language
}

\author{
Richard Candell \\ National Institute of Standards and Technology, \\ Gaithersburg, MD 20899, USA
}

richard.candell@nist.gov

Software DOI: https://doi.org/10.18434/T4/1502475

Software Version: 1.0

Key words: cyber-physical systems; factory communication; industrial communication; industrial control; industrial wireless; manufacturing; SysML; wireless sensor networks.

Accepted: October 15, 2018

Published: October 25, 2018

https://doi.org/10.6028/jres.123.018

\section{Summary}

Wireless technology is a key enabler of the vision of the future factory work-cell. Such a work-cell will operate autonomously with a high degree of mobility enabled by wireless technology. This paper describes the work-cell using the Systems Modeling Language (SysML) [1]. Using SysML the structural and parametric characteristics of the work-cell are described. Our model provides the architectural components and performance constraints of the work-cell in which wireless is used for a significant portion of connectivity. It identifies the structural components, interfaces, and data flows. Parametric characteristics that impact work-cell performance are included in the model. Using this model, industrial wireless networking requirements and work-cell behaviors may be developed and performance limits may be evaluated [2]. The SysML model presented here is developed using MagicDraw ${ }^{1} 18.5$ by No Magic [3].

\section{Software Specifications}

The software specifications for the SysML model are provided below:

\begin{tabular}{ll}
\hline NIST Operating Unit(s) & Engineering Laboratory, Intelligent Systems Division \\
\hline Category & Systems engineering model \\
\hline
\end{tabular}

${ }^{1}$ Certain commercial equipment, instruments, or materials are identified in this paper to specify the experimental procedure adequately. Such identification is not intended to imply recommendation or endorsement by the National Institute of Standards and Technology, nor is it intended to imply that the materials or equipment identified are necessarily the best available for the purpose. 


\begin{tabular}{ll}
\hline Targeted Users & Engineers, Researchers \\
\hline Operating System(s) & N/A \\
\hline Programming Language & SysML \\
\hline Inputs/Outputs & N/A \\
\hline & The full model and reports may be found in GitHub: \\
& https:/github.com/usnistgov/wireless-factory-sysml \\
& This software data item includes Extensible Markup Language (XML) \\
model and Hypertext Markup Language (HTML) report. MagicDraw 18.5 \\
or later with SysML plugin is required to edit the model. \\
Documentation & $\begin{array}{l}\text { The HTML documentation is provided for readers without the MagicDraw } \\
\text { software. }\end{array}$ \\
\hline Accessibility & N/A \\
\hline Disclaimer & https://www.nist.gov/director/licensing \\
\hline
\end{tabular}

\section{Problem Statement}

Constructing a factory work-cell that uses wireless technology as the primary mode of communication can be a daunting challenge. While wireless can bring many benefits to the industrial landscape to include untethered operation, lower cost, and increased operation visibility, it also has many challenges. For example, wireless communication uses a physical medium (i.e., the air interface) that is finite whereas wired communication solutions provide seemingly limitless bandwidth constrained only by the electronics on each end of the cable. Moreover, the wireless medium is an open natural resource available for any user regardless of the legalities that govern its use. This brings the possibility of interference, jamming, and friendly access competition. If wireless communication is used for mission critical operation such as safety or feedback control, then the impacts of this lossy medium on the operational system should be well understood. For this reason, a comprehensive model of the factory operation is required to project the impacts of wireless communication performance onto factory performance. The model should be easy to understand and manipulate for analytical purposes. The semantics of the language should be rich enough to convey architecture, behavior, and performance constraints, as well as be capable of supporting validation through integration with a simulation tool. This model addresses the problem of design capture and capability of validation through simulation.

\section{Model Implementation}

\subsection{Implementation Tool}

The SysML model was implemented using a proprietary graphical modeling tool called MagicDraw. MagicDraw supports the Unified Modeling Language (UML) and SysML among other graphic modeling languages.

\subsection{SysML Diagram Types}

The following SysML diagrams are used for communicating block relationships and architecture:

- Package Definition Diagram (PKG) - used to capture package containment and relationships;

- Block Definition Diagram (BDD) - used for capturing blocks, interfaces, and their relationships;

- Internal Block Diagram (IBD) - used for capturing the internal composition of a single block. 


\section{Conclusion}

A SysML model is developed to describe the architecture, behavior, and limitations of the wireless factory work-cell. The model is provided as a MagicDraw implementation and is available for public download at https://github.com/usnistgov/wireless-factory-sysml [4]. The model is accompanied by an HTML report that may be viewed using Internet browsers that support active content.

\section{References}

[1] Object Management Group (2017) OMG Systems Modeling Language, v1.5.

[2] Friedenthal S, Moore A, Steiner R (2015) A Practical Guide to SysML: The Systems Modeling Language (Elsevier/Morgan Kaufmann, Waltham, MA), Third Ed.

[3] No Magic (2018) MagicDraw SysML Software.

[4] Candell R (2018) A SysML Model of the Wireless Factory Work-cell. https://doi.org/10.18434/T4/1502475

About the author: Richard Candell is an Electronics Engineer in the Intelligent Systems Division at NIST. He leads a research project in wireless networks used for industrial control applications. The National Institute of Standards and Technology is an agency of the U.S. Department of Commerce. 